\title{
Venous Insufficiency in Pediatric Patients
}

\author{
(1) Melike Elif TEKER AÇIKEL¹, (D) Bekir INAN²
}

1University of Health Sciences, Haseki Training and Research Hospital, Clinic of Cardiovascular Surgery, İstanbul, Turkey

${ }^{2}$ Bezmialem Vakif University Faculty of Medicine, Department of Cardiovascular Surgery, İstanbul, Turkey

\begin{abstract}
Objective: The diagnosis and treatment of chronic venous disease has been well standardized in adults. However, the diagnosis of chronic venous insufficiency in pediatric patients, except for Klippel-Trénaunay syndrome and post-thrombotic syndrome, could not be established.

In this retrospective study, we planned to present pediatric patients diagnosed with venous insufficiency by our clinic.

Methods: Between January 2016 and May 2018, patients under 18 years of age who were referred to our clinic were included in this study. Venous Doppler ultrasonography was performed in patients and reflux in deep and superficial veins was evaluated. The reflux time is simply duration of inverse flow. A reflux time of $>500 \mathrm{~ms}$ was used to define the valve insufficiency of the superficial and perforating veins. A reflux time of $>1 \mathrm{~s}$ was used to define the valve insufficiency of the deep venous system. Venous reflux parameters were compared using t-test.

Results: Twenty-five patients were identified in this study. The mean age of the patients was 12 (4-17) years. As a result of clinical examination, 11 patients had varicose venous or venous malformation, 9 patients had swelling (pedal edema), and 6 patients had edema or venous ulcer. Two patients had normal examination findings. Chronic inflammation, hyperpigmentation was not present. No venous thrombomboemboli story was available in any patient.

Conclusion: The uncertainty of the factors that cause venous reflux in children still maintains itself today. However, this uncertainty will continue for a long time as the methods used in the diagnosis and treatment of chronic venous disease are the same in both adults and children.
\end{abstract}

Keywords: Child patients, venous insufficiency, Doppler ultrason, tromboemboli

\section{Introduction}

The diagnosis and treatment of chronic venous disease are well standardized in adults. However, the diagnosis of chronic venous insufficiency has not been established except in KlippelTrénaunay syndrome (KTS) and post-thrombotic syndrome in pediatric patients (1).

Because venous Doppler ultrasonography (RDUS) is used less in children than in adults and it is performed or interpreted according to adult protocol, it may cause less data on venous disease in pediatric patients (2).
In this retrospective study, we planned to present pediatric patients diagnosed with venous insufficiency by our clinic.

\section{Methods}

Patients under 18 years of age who were consulted to our clinic between January 2016 and May 2018 were included in this study. Demographic history, clinical examination and RDUS results of the patients were the indications for our study. Since our study was a retrospective study, informed consent was not obtained from the patients. As it was a retrospective study, ethics committee was not applied.

Address for Correspondence: Melike Elif TEKER AÇIKEL, University of Health Sciences Haseki Training 
RDUS was performed in the upright position as allowed by the patient (mobility, difficulty in positioning). Alternatively, patients with difficulty in that position were given supine position. All proximal veins were examined, including femoral vein and popliteal vein. Superficial vessels, including great saphenous veins, were evaluated at 3 to $5 \mathrm{~cm}$ intervals in a similar manner to accessory saphenous veins with small saphenous vein and perforating veins.

Reflux was evaluated in deep and superficial veins by venous Doppler ultrasonography. Reflux was achieved using a Valsalva maneuver for the common femoral vein or saphenofemoral junction by manual compression and release of the extremity until examination.

The reflux time was considered as the reverse flow time of the blood flow. A reflux time of $>500 \mathrm{~ms}$ was used to identify valve failure of the superficial and perforating veins. A reverse flow time of $>1$ second was used to define valve failure of the deep venous system (eg. common femoral, femoral, and popliteal veins).

Venous reflux parameters were compared by using variance analysis and unpaired t-test.

\section{Results}

A total of 25 patients were identified in this study. The mean age of the patients was 12 (4-17) years. Twelve of our patients had swelling in the foot, 9 had varicose veins, and 4 had redness/ venous ulcers in the foot (Table 1).

As a result of clinical examination performed on the patients, it was detected that 10 patients had lesions showing varicose vein or venous malformation, 9 patients had swelling (cases of pedal edema), and 4 patients had edema or venous ulcer accompanying to edema (one of which was pedal wounds consistent with advanced complex regional pain syndrome). Two patients had normal examination findings. There was no chronic inflammation or hyperpigmentation. None of the patients had a history of venous thromboembolism (Table 2).

In 11 of 18 patients who had venous reflux detected via venous Doppler ultrasonography, atypical triad such as capillary malformations (port wine stain), bone hypertrophy or lower extremity hypertrophy was observed to occur in addition to the diagnosis of chronic venous disease, and KTS was diagnosed.

Four of the patients with chronic venous insufficiency $(\mathrm{n}=6)$ specific to KTS had C2 disease (varicosity) and two had C3 disease (edema). One of the patients with chronic venous disease (CVD) and KTS ( $\mathrm{n}=3)$ had spider telangiectasia (C1), one patient had isolated varices (C2) and one had swelling with varicoses (C3). There were no patients with advanced venous disease (ie. C4-C6). One patient was classified as normal (C0) except very mild deep venous reflux (Table 3 ).

Postural orthostatic hypotension syndrome was found in 2 patients, vascular malformation affecting the lateral foot and posterolateral proximal calf and distal thigh in 2 patients, grade
Table 1. Demostrative data of patients

\begin{tabular}{l|l} 
Number of patients & 25 \\
\hline $\begin{array}{l}\text { Age (mean) } \\
\text { Symptoms }\end{array}$ & $12(4-17)$ years \\
$\begin{array}{l}\text { Swelling on foot } \\
\text { Varicose veins }\end{array}$ & 12 \\
\hline Redness/venous ulcer & 9 \\
\hline
\end{tabular}

Table 3. CEAP classification of patients

Patients with chronic venous insufficiency specific to Klippel-Trénaunay syndrome

\begin{tabular}{|c|c|c|}
\hline $\mathrm{C} 2$ & 4 & $6636 \%$ \\
\hline C3 & 2 & $33,3 \%$ \\
\hline $\begin{array}{l}\text { Patients with chronic venous insufficiency } \\
\text { and Klippel-Trénaunay Syndrome }\end{array}$ & 3 & $100 \%$ \\
\hline $\mathrm{C} 1$ & 1 & $33,3 \%$ \\
\hline C2 & 1 & $33,3 \%$ \\
\hline C3 & 1 & $33,3 \%$ \\
\hline C4-C6 & 0 & 0 \\
\hline CO & 0 & 0 \\
\hline
\end{tabular}

Table 2. Clinical examination results of patients

\begin{tabular}{|l|l|l|}
\hline Total number of patients & 25 & $100 \%$ \\
\hline Varicose vein or venous malformation & 10 & $40 \%$ \\
\hline Edema (pedal edema cases) & 9 & $36 \%$ \\
\hline Edema or accompanying venous ulcer & 4 & $16 \%$ \\
\hline Normal examination findings & 2 & $8 \%$ \\
\hline Chronic inflammation, hyperpigmentation & 0 & 0 \\
Venous thromboembolism & 0 & 0
\end{tabular}

I congenital lymphedema in 2 patients, complex regional pain syndrome in 1 patient, and acrocyanosis in 1 patient, and alternative diagnostic methods such as magnetic resorance, venography or lymphography were used.

The maximum great saphenous vein (GSV) diameter, reflux time, and valve closure rate were then compared to isolated patients, such as CVD, KTS, or an alternative diagnosis. The first observed mean GSV diameter was $0.52 \mathrm{~cm}$ and there was no significant difference in maximum GSV diameter between the groups.

\section{Discussion}

In this study, ten patients had varicose vein or lesions showing venous malformation, nine patients had swelling (pedal edema cases), and four patients had edema or edema accompanied by venous ulcers (one of which was consistent with advanced complex regional pain syndrome). Two patients had normal 
examination findings. There was no chronic inflammation or hyperpigmentation. None of the patients had a history of venous thromboembolism.

Although the diagnosis and treatment of CVD are well standardized in adults, no enough regulation could be made in children due to lack of data (1).

The Bochum Study (3) was initially performed for venous reflux on the development of venous disease in children aged between 10 and 12 years. According to this study, Although the incidence of venous disease in school-age children is $0.2-2.9 \%$ and the incidence of physiological venous reflux is approximately $13 \%$ in children aged between 14 and 16 years, RDUS has been used less in children than in adults $(3,4)$.

RDUS is the most important diagnostic method that can help us to answer all questions in patients with suspected venous insufficiency and Van Bemmelen et al. (5) showed in the late 1980 s that it could be safely used for the diagnosis of varices and venous insufficiency. RDUS evaluates both anatomical detail and hemodynamic changes in blood flow.

With RDUS, the presence and severity of reflux, vein diameters and the presence of obstruction in all three components of the venous system can be determined (6). We performed RDUS on our patients and evaluated the presence and severity of reflux, vein diameters and the existence of obstruction in the deep and superficial veins.

In our study, venous reflux was achieved by establishing a valsalva maneuver for the saphenofemoral junction by manual compression and release of the extremity until examination, as long as the patient's age or cooperation allowed.

The reflux time was considered as the reverse flow time of the blood flow. A reflux time of $>500 \mathrm{~ms}$ was used to identify valve failure of the superficial and perforating veins. A reverse flow time of $>1$ second was used to define the valve insufficiency of the deep venous system (eg. common femoral, femoral, and popliteal veins).

According to a conducted study, in spite of the presence of venous reflux in the duplex ultrasound imaging, signs and symptoms, $44 \%$ of children were diagnosed with an alternative disease consistent with venous disease. Our study was also consistent with this study. In another study, they found a $2.5 \%$ incidence of prevaginal saphenous reflux in school-age children (10-12 years) without evidence of venous varices by clinical examination and found that these children had a 30\% risk for the development of truncal varicose veins during their follow-ups (7). Truncal saphenous reflux was not observed in our patients.

Venous thromboembolism is a condition that can have serious consequences such as death in children (8). Complications of deep vein thrombosis (DVT), a form of venous thromboembolism, such as post-thrombotic syndrome and pulmonary embolism, may have significant long-term results although they occur less frequently in pediatric patients than in adult patients (9).
While $40-60 \%$ of children with venous thromboembolism may develop mild to moderate venous insufficiency symptoms, severe symptoms such as ulcers are very rare (10). Thrombosis in both iliofemoral veins and failure of venous recanalization have been identified as risk factors for post-thrombotic syndrome in children (10). None of our patients had thromboembolism.

KTS is a rare, sporadic, complex malformation characterized by (1) capillary malformations (port wine stain), (3) soft tissue and bone hypertrophy, or sometimes hypotrophy of a lower extremity and (4) atypical clinical triad. Mostly, lateral venous varices (11). Varicose veins or venous malformations are present in $72 \%$ of CTS patients (12). In our study, in 11 of the 18 patients who had venous reflux detected by venous doppler ultrasonography, atypical triad such as capillary malformations (port wine stain), bone hypertrophy or lower extremity hypertrophy was observed to coexist in addition to the diagnosis of chronic venous disease.

In recent screening studies, $10-15 \%$ of high school children have been found to have venous insufficiency symptoms. In our study, 3 of the patients diagnosed with venous insufficiency were in high school age. In a study conducted by Studennikova et al. (13), they found that the prevalence of chronic venous insufficiency increased in childhood and adolescence due to the relationship among genomic changes. In our study, genotypes of the patients could not be examined.

It has been suffered from the lack of diagnostic accuracy in chronic venous diseases for a long time, which has led to complex conclusions about the same disease in different studies. In 1994, at the American Venous Forum, classification and staging was performed in the CVD called CEAP (14). With this classification, clinical symptoms (C), etiologic (E) cause, anatomical features (A) and underlying pathophysiological events $(\mathrm{P})$ are defined (15). Clinical findings are divided into 6 stages according to the severity of the event: $\mathrm{C} 0=$ normal, $\mathrm{C} 1=$ spider/reticular veins, $\mathrm{C} 2=$ varicose veins, C3=edema, C4=skin changes, C5=healed ulcer and $\mathrm{C} 6=$ active ulcer $(16,17)$. We also divided the patients into groups using the CEAP classification.

In our study, 4 of the patients with chronic venous insufficiency $(\mathrm{n}=6)$ specific to KTS had C2 disease (varicosity) and two had C3 disease (edema). One of the patients with CVD and KTS $(\mathrm{n}=3)$ had spider telangiectasia $(\mathrm{C} 1)$, one patient had isolated varices (C2) and one had swelling with varicoses (C3). There were no patients with advanced venous disease (ie. C4-C6). We classified one patient as normal (C0) except for very mild deep venous reflux.

Nurmeev et al. (18) used the CEAP classification in their study and they reported that they encountered $\mathrm{C} 1$ class more frequently in children. Also, in our study, we found that $\mathrm{C} 1$ class was more common in children.

\section{Conclusion}

The uncertainty of the causative agents of venous reflux in children is still present. In addition, uncertainty will continue for a long time due to the determinative methods and treatment protocol applied to adult individuals. 


\section{Ethics}

Ethics Committee Approval: As it was a retrospective study, no ethics committee was applied.

Patient consent: Since our study was a retrospective study, consent was not obtained from the patients.

Reviewer evaluation: The study was evaluated by people outside the editorial board.

\section{Authors Contributions}

Concept: M.E.T.A., B.İ., Design: B.İ., Data collecting and processing: M.E.T.A., Analysis or interpretation: M.E.T.A., B.İ., Literature review: B.İ., Written by: M.E.T.A.

Conflict of Interest: Authors declare no conflict of interest.

Financial support: Authors declare that they received no financial support.

\section{Reference}

1. Gloviczki P, Comerota AJ, Dalsing MC, Eklof BG, Gillespie DL, Gloviczki ML, et al. The care of patients with varicose veins and associated chronic venous diseases: clinical practice guidelines of the Society for Vascular Surgery and the American Venous Forum. J Vasc Surg 2011;53:2-48.

2. Stapler SJ, Zurales K, Mazurek A, Otemuyiwa B, Knol M, Wakefield TW, et al. A pilot study of venous duplex ultrasound parameters in healthy children. J Vasc Surg Venous Lymphat Disord 2018;3:34750.

3. Schultz-Ehrenburg U, Reich-Schupke S, Robak-Pawelczyk B, Rudolph T, Moll C, Weindorf N, et al. Prospective epidemiological study on the beginning of varicose veins: Bochum Study I-IV. Phlebologie 2009;38:17-25.

4. Wright JM, Watts RG. Venous thromboembolism in pediatric patients: epidemiologic data from a pediatric tertiary care center in Alabama. J Pediatr Hematol Oncol 2011;33:261-4.

5. Van Bemmelen PS, Bedford G, Beach K, Strandness DE. Quantitative segmental evaluation of venous valvular reflux with duplex ultrasound scanning. J Vasc Surg 1989;10:425-31.
6. Coleridge-Smith P, Labropoulos N, Partsch H, Myers K, Nicolaides A, Cavezzi A. Duplex ultrasound investigation of the veins in chronic venous disease of the lower limbs-UIP consensus document. Part I. Basic principles. Eur J Vasc Endovasc Surg 2006;31:83-92.

7. Schultz-Ehrenburg U, Weindorf N, Matthes U, Hirche H. An epidemiologic study of the pathogenesis of varices. The Bochum study I-III. Phlebologie 1992;45:497-500.

8. Raffini L, Huang Y, Witmer C, Feudtner C. Dramatic increase in venous thromboembolism in children's hospitals in the United States from 2001-2007. Pediatrics 2009;124:1001-8.

9. Avila M, Pullenayegum E,Williams S, Yue N, Krol P, Brandao LR. Postthrombotic syndrome and other outcomes of lower extremity deep vein thrombosis in children. Blood 2016;128:1862-9.

10. Spentzouris G, Gasparis A, Scriven RJ, Lee TK, Labropoulos N. Natural history of deep vein thrombosis in children. Phlebology 2015;30:412-7.

11. Gloviczki P, Driscoll DJ. Klippel-Trénaunay syndrome: current management. Phlebology 2007;22:291-8.

12. Jacob AG, Driscoll DJ, Shaughnessy WJ, Stanson AW, Clay RP, Gloviczki P. Klippel-Trénaunay syndrome: spectrum and management. Mayo Clin Proc 1998;73:28-36.

13. Studennikova VV, Severgina LO, Dzyundzya AN, Korovin IA. Lower extremity varicose veins in childhood and at a young age: Mechanism of development and specific features. Arkh Patol 2017;79:56-60.

14. Padberg FT Jr. CEAP classification for chronic venous disease. Dis Mon 2005 51:176-82.

15. Porter JM, Moneta GL. Reporting standarts in venous disease:an update.International Consensus Commitee on chronic venous disease. J Vasc Surg 1995;21:635-45.

16. Teruya TH, Ballard JL. New approaches for the treatment of varicose veins. Surg Clin North Am 2004;84:1397-417.

17. Raju S, Neglén P. Clinical practice. Chronic venous insufficiency and varicose veins. N Engl J Med 2009;360:2319-27.

18. Nurmeev IN, Mirolubov LM, Mirolubov AL, Nurmeev NN, Osipov AY, Nurmeeva AR, et al. Treatment of chronic venous diseases in children and adolescents. Angiol Sosud Khir 2016;22:105-9. 